Article

\title{
Entanglement Entropy in a Triangular Billiard
}

\author{
Sijo K. Joseph * and Miguel A. F. Sanjuán
}

Nonlinear Dynamics, Chaos and Complex Systems Group, Departamento de Física, Universidad Rey Juan Carlos, Tulipán s/n, Móstoles, Madrid 28933, Spain; miguel.sanjuan@urjc.es

* Correspondence: sijo.joseph@urjc.es; Tel.: +34-914-88-71-61

Academic Editors: Gerardo Adesso and Kevin H. Knuth

Received: 4 November 2015; Accepted: 23 February 2016; Published: 1 March 2016

\begin{abstract}
The Schrödinger equation for a quantum particle in a two-dimensional triangular billiard can be written as the Helmholtz equation with a Dirichlet boundary condition. We numerically explore the quantum entanglement of the eigenfunctions of the triangle billiard and its relation to the irrationality of the triangular geometry. We also study the entanglement dynamics of the coherent state with its center chosen at the centroid of the different triangle configuration. Using the von Neumann entropy of entanglement, we quantify the quantum entanglement appearing in the eigenfunction of the triangular domain. We see a clear correspondence between the irrationality of the triangle and the average entanglement of the eigenfunctions. The entanglement dynamics of the coherent state shows a dependence on the geometry of the triangle. The effect of quantum squeezing on the coherent state is analyzed and it can be utilize to enhance or decrease the entanglement entropy in a triangular billiard.
\end{abstract}

Keywords: continuous-variable quantum entanglement; triangular billiard

\section{Introduction}

There are many interesting studies concerning the quantum and classical properties of the two-dimensional geometries like the Robnik billiard [1,2] and the Bunimovich stadium $[3,4]$. A quantum particle inside a triangular potential is another interesting problem studied by several researchers [5-9]. It was Krishnamurthy et al. [10] who found a transformation to map the three-particle collision problem in one dimension into the problem of a quantum particle inside a two-dimensional triangular domain. Interestingly, the triangular shaped potential can appear in several contexts, for example it can appear in the equipotental curves for the Hénon-Heiles system at the critical energy [11]. The exception in this case is that, inside the boundary the potential behaves like a two-dimensional harmonic oscillator. However, in the billiard case, the particle has a free motion inside the triangular shaped domain.

Casati and Prosen describe [7] three classes of triangular billiards: $(A)$ All angles are rational with $\pi,(B)$ Only one angle is rational with $\pi,(C)$ All angles are irrational with $\pi$. The dynamics of type $A$ triangles is not ergodic; in fact, it is pseudointegrable. Type $B$ triangles are generic right triangles which are ergodic and weakly mixing. Earlier, it was believed that triangular billiards were only weakly mixing. Casati and Prosen [7] provided the numerical evidences for strong mixing properties for the type $C$ triangles. Then it was shown in [8] that the irrational triangles may vary smoothly between the limits of strong mixing and regular behaviors. Furthermore, in [9] it was also shown that the level statistics of the irrational triangle indeed obey the Gaussian orthogonal ensemble (GOE) distribution and its intermediate statistics.

On the other hand, there is an increased interest in continuous-variable (CV) entanglement [12-15]. It is already known, both theoretically and experimentally, that the quantum entanglement can indeed detect the chaotic behavior appearing in the classical system [16-19]. 
Recently it is shown that, by changing the boundary domain from regular to chaotic, the quantum entanglement can be enhanced in a system [20]. Hence, the classical dynamical properties can be reflected in the properties of quantum entanglement and as consequence it can be used for the entanglement enhancement.

In this article, we make a brief review of the three-particle collision system in one dimension and its relation to the triangular billiard geometry. Then, we explain the quantum entanglement in the reduced two-particle system, which is identical to the single particle trapped in a two-dimensional triangular domain. The von Neumann entropy of entanglement is briefly explained, and we analyze the bipartite entanglement in the ground state eigenfunction. Similarly, we compute the average entanglement entropy with 500 initial eigenfunctions and relate the entanglement with the irrationality of the triangular geometry. We observe that the average entanglement entropy depends on the irrationality of the triangle. We also analyze the entanglement dynamics of the coherent state and squeezed coherent state with its center chosen at the centroid of the triangle.

\section{Model}

Let us consider the time independent Schrödinger equation for three hard core particles restricted to the domain $[0 \mathrm{~L}]$ with coordinates $\theta_{1}, \theta_{2}$ and $\theta_{3}$, which is given by,

$$
-\frac{\hbar^{2}}{2 m} \sum_{i=1}^{i=3} \frac{\partial^{2} \Psi}{\partial \theta_{i}^{2}}=E \Psi
$$

H. R. Krishnamurthy et al. [10] had found a transformation of $\theta_{i}$ variables into new variables $y_{i}$,

$$
\begin{aligned}
& y_{1}=\frac{1}{\sqrt{2}}\left(\theta_{1}-\theta_{2}\right) \\
& y_{2}=\sqrt{\frac{2}{3}}\left(\frac{1}{2}\left(\theta_{1}+\theta_{2}\right)-\theta_{3}\right) \\
& y_{3}=\frac{1}{\sqrt{3}}\left(\theta_{1}+\theta_{2}+\theta_{3}\right) .
\end{aligned}
$$

Then they have found that, without loss of generality, restricting the $\theta$-space into the region $\theta_{1} \geq \theta_{2}, \theta_{2} \geq \theta_{3}$ and $\theta_{3}+2 \pi \geq \theta_{1}$, we can transform the system into a two-dimensional Schrödinger equation in a triangular domain. In the transformed domain, the equilateral triangle is given on the $y_{1}-y_{2}$ plane, which is defined by $y_{1} \geq 0, y_{1} \leq \sqrt{3} y_{2}, \frac{y_{1}}{\sqrt{2}} \leq 2 \pi-\sqrt{\frac{3}{2}} y_{2}$. The corners of the equilateral triangle are given by, $(0,0),\left(\sqrt{2} \pi, \sqrt{\frac{2}{3}} \pi\right),\left(0,2 \sqrt{\frac{2}{3}} \pi\right)$ and the transformed Schrödinger equation can be written as,

$$
-\frac{\hbar^{2}}{2 m}\left(\frac{\partial}{\partial y_{1}^{2}}+\frac{\partial}{\partial y_{2}^{2}}\right) \Psi=E \Psi .
$$

Here the boundary condition is such that the wavefunction should vanish on the boundary of the equilateral triangle. Hence the eigenfunctions are given by,

$$
\Psi\left(y_{1}, y_{2}\right)_{m, n}=\left|\begin{array}{ccc}
1 & 1 & 1 \\
\exp \left(i \ell\left(\frac{y_{1}}{\sqrt{2}}+\frac{y_{2}}{\sqrt{6}}\right)\right) & \exp \left(i \ell\left(-\frac{y_{1}}{\sqrt{2}}+\frac{y_{2}}{\sqrt{6}}\right)\right) & \exp \left(-i \ell \frac{2 y_{2}}{\sqrt{6}}\right) \\
\exp \left(i m\left(\frac{y_{1}}{\sqrt{2}}+\frac{y_{2}}{\sqrt{6}}\right)\right) & \left.\exp \left(i m-\frac{y_{1}}{\sqrt{2}}+\frac{y_{2}}{\sqrt{6}}\right)\right) & \exp \left(-i m \frac{y_{2}}{\sqrt{6}}\right)
\end{array}\right| .
$$

The complete three-particle eigenfunction of our problem contains an additional phase factor $\exp i\left(n_{1}+n_{2}+n_{3}\right) \frac{y_{3}}{\sqrt{3}}$ due to the contribution from the third particle, but we can easily ignore it. Effectively the three-particle problem can be reduced into the two-particle problem. This analytical 
solution is valid only for particles with identical mass, and it corresponds to an equilateral triangle geometry, which is clearly a pseudointegrable situation. However, the eigenfunctions are not known for the case of a general triangular geometry. Hence, we analyze a more general case of the triangles. The two-dimensional Schrödinger equation (Equation (5) can be written in terms of the Helmholtz equation with a Dirichlet boundary condition,

$$
\begin{aligned}
\left(\nabla^{2}+k^{2}\right) \Psi\left(y_{1}, y_{2}\right) & =0, \text { for }\left(y_{1}, y_{2}\right) \text { in } D \\
\Psi\left(y_{1}, y_{2}\right) & =0 \text {, for }\left(y_{1}, y_{2}\right) \text { on } \partial D
\end{aligned}
$$

where $D$ is the triangular domain, $\partial D$ defines its boundary and $k^{2}=2 m E / \hbar^{2}$. Generalizing our geometry from the simple equilateral triangle to the irrational triangle, the boundary of the domain $\partial D$ can be specified by any two of the angles $\alpha, \beta, \gamma$ and the area $\mathcal{A}$. Since the area $\mathcal{A}$ is just a scaling factor we can ignore it by properly scaling the energy eigenvalues.

As we have discussed earlier, the motion of a point particle inside a triangular billiard is equivalent to the motion of two point particles interacting only through elastic collisions. It can be seen that the mass ratios of the particle determine the angle $\alpha, \beta$ and $\gamma$ of the corresponding triangle via the relation

$$
\begin{aligned}
\tan \alpha & =\sqrt{\frac{m_{2} M}{m_{1} m_{3}}}, \\
\tan \beta & =\sqrt{\frac{m_{1} M}{m_{2} m_{3}}}, \\
\tan \gamma & =\sqrt{\frac{m_{3} M}{m_{1} m_{2}}},
\end{aligned}
$$

where $m_{1}, m_{2}, m_{3}$ are the mass of each particle and $M=m_{1}+m_{2}+m_{2}$, which is the total mass of the three-particle system [7]. Considering the system with identical mass $m_{1}=m_{2}=m_{3}$, the equilateral triangle domain can be recovered. Hence changing the geometry of the triangle has a physical meaning associated to it, which is physically equivalent to considering different mass ratios. In the usual sense, building triangular billiards can be done easily by assigning rational or irrational values to the ratio between the inner angles of the triangle and $\pi$. Here, we follow the approach used by F. M. de Aguiar et al. [8,9]. In order to construct the irrational triangular billiard, he has considered acute triangles with sides $N, N+1$, and $N+2$, where $N$ is an integer. This scheme has the advantage that each triangle can be solely identified with the parameter $N$. According to the theorem given in [21], if $q \in Q$ with $0 \leq q \leq 1$, then, the number $\cos ^{-1} \sqrt{q} / \pi$ is rational if and only if $q$ is $0,1 / 4,1 / 2,3 / 4$, or 1 . As a corollary [8], it can be observed that the triangles with sides given by consecutive integers $(N, N+1, N+2)$ have all angles irrational with $\pi$ if $3 \leq N \leq \infty$. It is well known that $(3,4,5)$ is a Pythagorean triple which implies that at $N=3$ we have a right triangle. In our numerical analysis, we start with $N=3$ until the value $N=53$. For the higher values of $N$ the triangle gradually approaches to an equilateral one, and at $N=\infty$ we get an equilateral triangle .

\section{Von Neumann Entropy of Entanglement of the Triangular Eigenfunctions}

It is worthwhile to explore the geometrical dependence of entanglement in the eigenfunctions of the triangular billiard and this is still an unexplored topic. Here we analyze the bipartite entanglement appearing in the two-dimensional eigenfunction. The particles can be entangled due to the fact that they interact via collisions. The nature of their collision interaction is reflected in the angles of the triangular billiard. We take $y_{1}$ as the horizontal $x$-axis and $y_{2}$ as the $y$-axis. The entanglement between the wave function of $y_{1}$ and $y_{2}$ variables can be easily studied, the wave function associated to $y_{1}$ and $y_{2}$ corresponds to the quantum mechanical state of particle 1 and particle 2 . Usually the entanglement 
can be measured either using the linear entropy of entanglement, entanglement witness, concurrence, logarithmic negativity etc. [13,22]. In order to explore the continuous variable bipartite entanglement, we are using here the von Neumann entanglement entropy.

Now we need to evaluate the reduced density function using the wavefunction $\Psi\left(y_{1}, y_{2}\right)$, in order to compute the continuous variable entanglement in $y_{1}$ and $y_{2}$ variable. The reduced density function of the first subsystem $\rho_{1}$ can be obtained by integrating over the second particle mode, and it can be represented in terms of the bipartite wave function $\Psi\left(y_{1}, y_{2}\right)$, i.e.,

$$
\rho_{1}\left(y_{1}, z_{1}\right)=\int \Psi\left(y_{1}, y_{2}\right) \Psi^{*}\left(z_{1}, y_{2}\right) d y_{2},
$$

where $\rho_{1}(x, z)$ is the reduced density function of the first subsystem in the continuous position basis representation. To quantify the entanglement, we use the entanglement entropy defined as the von Neumann entropy of the reduced density matrix:

$$
S_{v n}(t)=-\operatorname{Tr}\left(\rho_{1} \log \rho_{1}\right) .
$$

Similarly, the von Neumann entropy of entanglement can be written in terms of the eigenvalues of the reduced density matrix which is given by,

$$
S_{v n}(t)=-\sum_{i} \lambda_{i} \log \lambda_{i}
$$

where $\lambda_{i}$ are the eigenvalues of the Hermitian kernel $\rho_{1}\left(y_{1}, z_{1}\right)$. These eigenvalues are numerically computed from the Fredholm type I integral equation of $\rho_{1}\left(y_{1}, z_{1}\right)$, which is given by

$$
\int \rho_{1}\left(y_{1}, z_{1}\right) \phi_{i}\left(z_{1}\right) d z_{1}=\lambda_{i} \phi_{i}\left(y_{1}\right)
$$

where $\lambda_{i}$ are the eigenvalues with the corresponding Schmidt eigenfunctions $\phi_{i}(x)$. On the discretized domain this Schmidt eigenvalue equation can be easily transformed into the matrix eigenvalue problem, and the diagonalization of the kernel can yield the Schmidt eigenvalues.

\section{Geometric Dependence of Entanglement and the Irrationality of the Triangle}

The relationship between a quantum chaotic geometry and entanglement is already explored in different works. The triangular billiard exhibit a peculiar pseudochaotic property which is entirely different from the normal quantum chaotic billiards. It is well known that there are different stages of ergodic regime called the ergodic hierarchy. Ergodic hierarchy provides a hierarchy of increasing degrees of randomness in a system and it is useful in characterising the behaviour of Hamiltonian dynamical systems [23]. It typically consists of five levels: Sheer Ergodic $\supset$ Weak Mixing $\supset$ Strong Mixing $\supset$ Kolmogorov $\supset$ Bernoulli. Positive K-S entropy or the chaotic property is assured only for the Kolmogorov and Bernoulli systems while pseudochaos is a partiular case of weak chaos with zero K-S entropy. In $[8,9]$, it has been shown that the irrational triangular billiard can indeed exhibit the pseudochaotic property. Taking this into account, we would like to explore how the irrationality of a triangle or the pseudochaotic can affect the bipartite entanglement of the eigenmodes.

In order to compute the eigenfunctions, we have taken the $(N, N+1, N+2)$ sided triangle in the $y_{1}-y_{2}$ plane. The orientation of the triangle is chosen in such a way that the side $N+2$ always lies along the horizontal $y_{1}$ axis. After properly defining the triangular domain, we have computed the Hamiltonian matrix of the system and we diagonalized it to obtain the eigenfunctions. In Figure 1, we have computed the ground state eigenfunctions of the irrational triangle billiard. Figure 1a-d show the irrational triangles with numbers $N=3, N=13, N=23$ and $N=33$, respectively. It can be easily seen that, the first triangle shown in Figure 1a is a right triangle, and the triangle shown in Figure 1d is an approximation of an equilateral triangle, where Figure $1 b, c$ show intermediate geometries. 


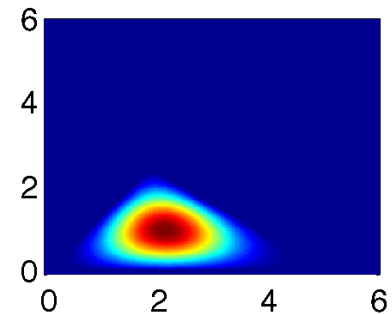

(a)

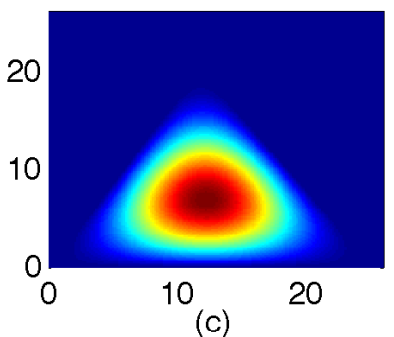

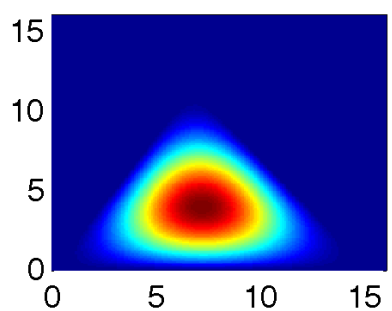

(b)

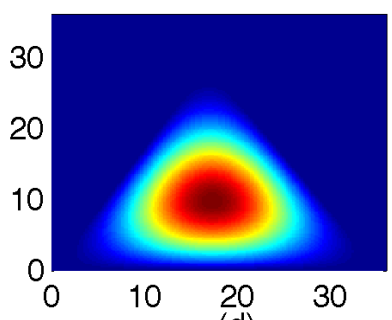

(d)

Figure 1. The ground state eigenfunctions of the different irrational triangles are shown. Figure 1a-d show the irrational triangles with numbers $N=3, N=13, N=23$ and $N=33$, respectively. It can be easily seen that, the first triangle shown in (a) is a right triangle and the triangle shown in (d) is approximate to an equilateral triangle, where (b) and (c) show intermediate geometries.

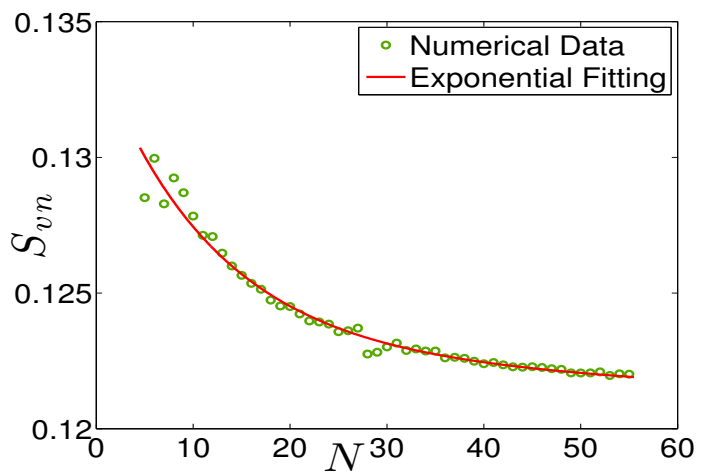

(a)

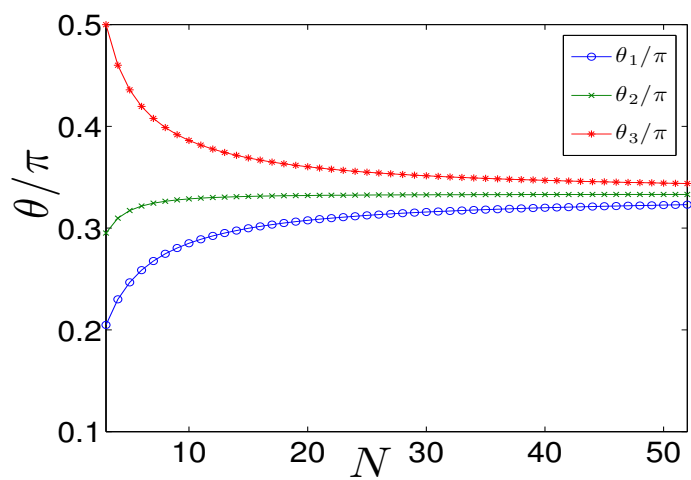

(b)

Figure 2. In (a) the von Neumann entropy of entanglement $S_{v n}$ for the ground state eigenfunctions of the irrational triangles is plotted against the different triangle configurations. It can be easily seen that in both cases the entanglement entropy reduces as we increase $N$ or as we approach the equilateral triangle. Red curve shows the exponential fit of data, where the equation of the curve is given by $S_{v n}=A e^{-\alpha N}+\beta$, where $A=0.01123, \alpha=0.08247$ and $\beta=0.1227$. In (b) the angles of the triangle $\theta_{1}, \theta_{2}$ and $\theta_{3}$ are plotted for different triangle configurations and it can be seen that as $\mathrm{N}$ grows, the angles $(\theta / \pi)$ approaches the rational value of $1 / 3$.

In Figure 2a we have plotted the von Neumann entanglement entropy $S_{v n}$ for the ground state eigenfunctions by varying the value of the parameter $N$. We slowly increase $N$ from 3 to 53 , and it is well known that the first triangle is Pythagorean, and as we increase $N$, it can approach to an equilateral triangle. As we have already discussed, F. M. de Aguiar [8] had already analyzed the irrationality of the triangle and he has found that as we increase $N$ the irrationality reduces except for the fact that it slightly increases until the triangle around $N=10$, then it drops to smaller values as we increase $N$. In order to compute the value of the entanglement for the equilateral triangle, or as the limit $N \rightarrow \infty$ we have made a scaling analysis of the entanglement entropy $S_{v n}$. The 
ground state entanglement entropy is approximated by the red curve $S_{v n}=A e^{-\alpha N}+\beta$ and the parameters are determined as $A=0.01123, \alpha=0.08247$ and $\beta=0.1227$ respectively. From the equation $S_{v n}=A e^{-\alpha N}+\beta$, it can be seen that, as $N \rightarrow \infty$ the asymptotic value of the entanglement entropy $S_{v n} \rightarrow \beta$ which is found to be $S_{v n}=0.1227$.

In Figure $2 \mathrm{~b}$, we have plotted the three angles $\theta_{1}, \theta_{2}$ and $\theta_{3}$ of the triangle as a function of the number $N$. As the triangle approaches an equilateral one, the angle approaches the rational ratio $1 / 3$ with respect to $\pi$.

We have plotted the first 500th excited state eigenfunction of the irrational triangle billiard in Figure 3. Here Figure 3a-d show the irrational triangles with numbers $N=3, N=13, N=23$ and $N=33$, respectively. In order to have a clear picture, in Figure 4, we have plotted the average von Neumann entanglement entropy $\bar{S}_{v n}$ for the lowest 500 eigenfunctions. From Figure 4, it can be easily seen that the average entanglement computed from the first 500 eigenfunctions increases until $N=10$ and later it starts decreasing. In [8], author had studied the relationship between strong mixing and irrationality of the triangular geometry. He has found that the strong mixing is indeed related to the irrationality of the triangle and even the level statistics are Gaussian orthogonal ensemble (GOE) distribution and its intermediate statistics. Note that the system is strongly mixing, while there is no chaos in the system. In [8], they have shown that the irrationality of the triangle is maximum at $N=10$, then it reduces gradually as it approaches to an equilateral triangle. The average entanglement entropy follows the same pattern which in accordance with the irrationality measure of the triangle shown in [8]. Hence, we see a correspondence between the bipartite entanglement and the irrationality of the triangle geometry.

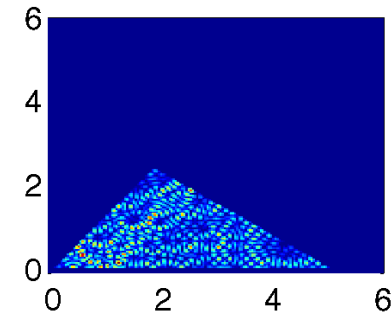

(a)

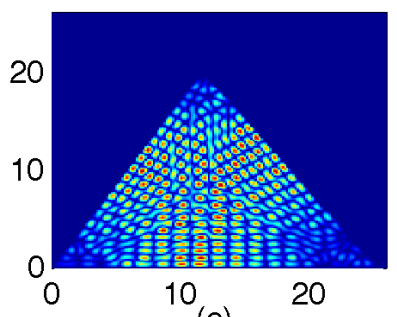

(c)

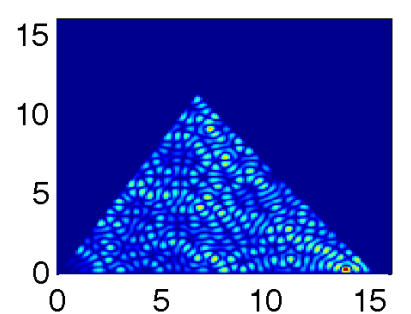

(b)

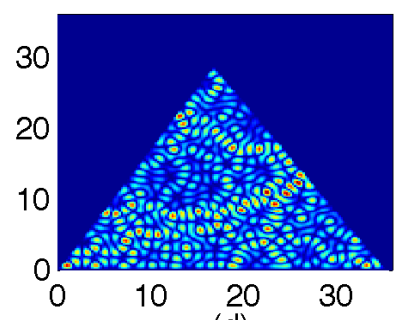

(d)

Figure 3. The 500th excited eigenfunctions of the different irrational triangles are shown in the figure. It is the highest eigenfunction utilized to compute the average von Neumann entropy of entanglement. Figure 3a-d show the irrational triangles with numbers $N=3, N=13, N=23$ and $N=33$, respectively. It can be easily seen that, the first triangle shown in (a) is a right triangle and the triangle shown in $(\mathbf{d})$ is approximately closer to an equilateral triangle, where (b) and (c) show intermediate ones. 


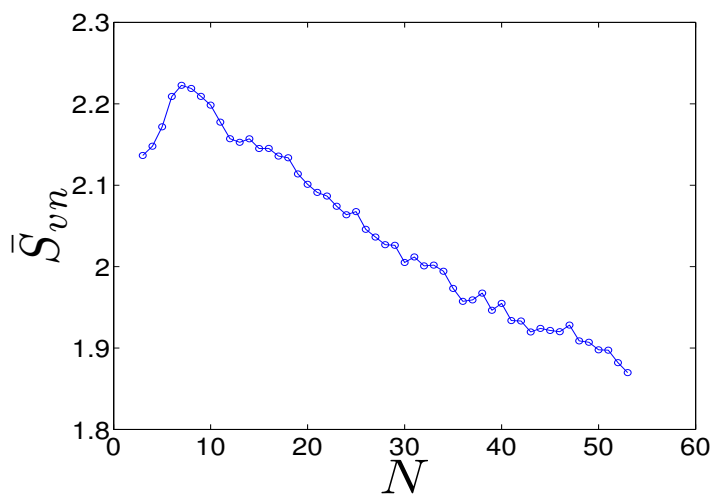

Figure 4. The average von Neumann entropy of entanglement $\bar{S}_{v n}$ is plotted against the different triangle configurations. It can be easily seen that the entanglement entropy reduces as we increase $N$ or as we approach the equilateral triangle.

\section{Entanglement Entropy for the Coherent States and the Squeezed Coherent States}

It is worthwhile to explore the time evolution of the entanglement entropy for a tensor product coherent state or the squeezed coherent state inside a triangular billiard. One may wonder about the validity of choosing the harmonic oscillator coherent state or the squeezed coherent state inside the triangular billiard. Recently, it has gotten the attention to the fact that the chaotic billiard geometries can indeed be used for the optical fiber cross-section [24,25]. Hence it has practical applications concerning the light propagation inside a triangular shaped fiber. It has been also shown that the chaotic billiard geometry can be exploited to generate the classical entanglement in the transverse modes of a classical electromagnetic field inside an optical fiber [20].

In order to compute the entanglement entropy for the coherent state for different triangular geometries, it is necessary to fix the center of the wave-packet for different triangular geometries. For that purpose, we have chosen the centroid of each triangle as the location of the center of the wave-packet. It is well known that the centroid or geometric center of the triangle can be easily found by knowing the coordinates of the its vertices. The arithmetic mean position of all the three vertex points will give the coordinate of the centroid of the triangle. Since we consider the triangles with sides $(N, N+1, N+2)$, the angles can be easily computed using the law of cosines. Since we take the $N+2$ side horizontal to the $x$-axis, hence the two vertices $(0,0)$ and $(N+2,0)$ are known. We only need to know the two base angles or the slope with respect to the side $N+2$ to determine the triangle. The point of intersection of the two lines with slope $m_{1}$ and $m_{2}$ is used to determine one of the missing vertex. The equation of the first line is $y_{1}=n_{1} x_{1}$, while the equation of the second line is given by $y_{2}=n_{2}\left(x_{2}-(N+2)\right)$. Hence the coordinate of the third vertex is computed by finding the intersection of both lines and it is given by,

$$
\begin{aligned}
& x_{v_{3}}=\frac{n_{2}(N+2)}{\left(n_{2}-n_{1}\right)}, \\
& y_{v_{3}}=\frac{n_{1} n_{2}(N+2)}{\left.\left(n_{2}-n_{1}\right)\right)} .
\end{aligned}
$$

Hence, the coordinate centroid of the triangle $\left(x_{c}, y_{c}\right)$ for a given number $N$ is given by,

$$
\begin{aligned}
& x_{c}=\frac{n_{2}(N+2)}{3\left(n_{2}-n_{1}\right)}, \\
& y_{c}=\frac{(N+2)}{3}\left(1+\frac{n_{1} n_{2}}{\left(n_{2}-n_{1}\right)}\right) .
\end{aligned}
$$


For our investigation, we use the Hollenhorst and Caves definition of the squeezing operator [26-28] and we employ the coordinate representation of the squeezed coherent state [29] for the numerical computations. The squeezed coherent state is defined as

$$
\left|\alpha_{k}, \zeta_{k}\right\rangle=\hat{D}\left(\alpha_{k}\right) \hat{S}\left(\zeta_{k}\right)|0\rangle,
$$

where the displacement operator $\hat{D}$ and the squeezing operator $\hat{S}$ is given by

$$
\begin{aligned}
\hat{D}\left(\alpha_{k}\right) & =\exp \left(\alpha_{k}{\hat{a_{k}}}^{\dagger}-\alpha_{k}^{*} \hat{a_{k}}\right), \\
\hat{S}\left(\zeta_{k}\right) & =\exp \left(\frac{1}{2} \zeta_{k}{\hat{a_{k}}}^{{ }^{2}}-\frac{1}{2} \zeta_{k}{ }^{*}{\hat{a_{k}}}^{2}\right),
\end{aligned}
$$

and $\alpha_{k}=\left|\alpha_{k}\right| e^{\mathrm{i} \phi_{k}}, \zeta_{k}=\left|r_{k}\right| e^{\mathrm{i} \theta_{k}}$ are complex numbers and $\alpha_{k}$ are related to the phase space variables $\left(q_{k}, p_{k}\right)$ in the following manner

$$
\alpha_{k}=\frac{1}{\sqrt{2 \hbar}}\left(q_{k}+\mathrm{i} p_{k}\right)
$$

with $k=1,2$, respectively. According to Møller et al. [29], the squeezed coherent state in the $\mathbf{x}$ position basis can be written as

$$
\begin{gathered}
\psi\left(\mathbf{x}, \alpha_{k}, \zeta_{k}\right)=\left(\frac{1}{\pi \hbar}\right)^{1 / 4}\left(\cosh r_{k}+\mathrm{e}^{\mathrm{i} \theta} \sinh r_{k}\right)^{-1 / 2} \\
\exp \left\{-\frac{1}{2 \hbar}\left(\frac{\cosh r_{k}-\mathrm{e}^{\mathrm{i} \theta} \sinh r_{k}}{\cosh r_{k}+\mathrm{e}^{\mathrm{i} \theta} \sinh r_{k}}\right)\left(\mathbf{x}-q_{1}\right)^{2}+\frac{\mathrm{i}}{\hbar} p_{1}\left(\mathbf{x}-q_{1} / 2\right)\right\} .
\end{gathered}
$$

The tensor product state of this wavefunction is used to study the classical entanglement dynamics for different squeezing parameter values. Since $q_{1}$ and $q_{2}$ denote the center of the position coordinate of the coherent state, we can fix it as the centroid of the triangular billiard $x_{c}$ and $y_{c}$. For the lowest energy case, we fix $p_{1}=0$ and $p_{2}=0$. Hence the tensor product coherent state can be written as,

$$
\begin{gathered}
\Psi\left(y_{1}, y_{2}\right)=\left(\frac{1}{\pi \hbar}\right)^{1 / 2}\left(\cosh r_{1}+\mathrm{e}^{\mathrm{i} \theta} \sinh r_{1}\right)^{-1 / 2}\left(\cosh r_{2}+\mathrm{e}^{\mathrm{i} \theta} \sinh r_{2}\right)^{-1 / 2} \\
\exp \left\{-\frac{1}{2 \hbar}\left(\frac{\cosh r_{1}-\mathrm{e}^{\mathrm{i} \theta} \sinh r_{1}}{\cosh r_{1}+\mathrm{e}^{\mathrm{i} \theta} \sinh r_{1}}\right)\left(y_{1}-x_{c}\right)^{2}\right\} \times \exp \left\{-\frac{1}{2 \hbar}\left(\frac{\cosh r_{2}-\mathrm{e}^{\mathrm{i} \theta} \sinh r_{2}}{\cosh r_{2}+\mathrm{e}^{\mathrm{i} \theta} \sinh r_{2}}\right)\left(y_{2}-y_{c}\right)^{2}\right\},
\end{gathered}
$$

where $x_{c}$ and $y_{c}$ are given in Equations (17) and (18) respectively. The time evolution of the squeezed coherent state is performed via the unitary operator $U(t)=\exp \left(\frac{-i t}{\hbar} \hat{H}\right)$, where $\hat{H}$ is the Hamiltonian of the system. The wavefunction at any instant $t$ is computed via $\Psi(x, y, t)=U(t) \Psi(x, y)$ and the time evolution of the entanglement entropy $S_{v n}(t)$ can be easily computed using Equations (11) and (13).

We have plotted in Figure 5 the entanglement entropy $S_{v n}(t)$ for different triangle configurations $N=3,4,5,6$ respectively. The initial coherent state is chosen at the centroid of the each triangle and the squeezing parameters are taken as zero and the Planck constant $\hbar=0.025$. It can be seen that during the time evolution of the wave packet, the initial tensor product state becomes entangled due to the collision with the wall of the triangle. A small value of the Planck constant is chosen in such a way that the wave packet fit inside in the triangular billiard. It can be easily seen that, as we change the geometry of the triangle the entanglement dynamics changes. 


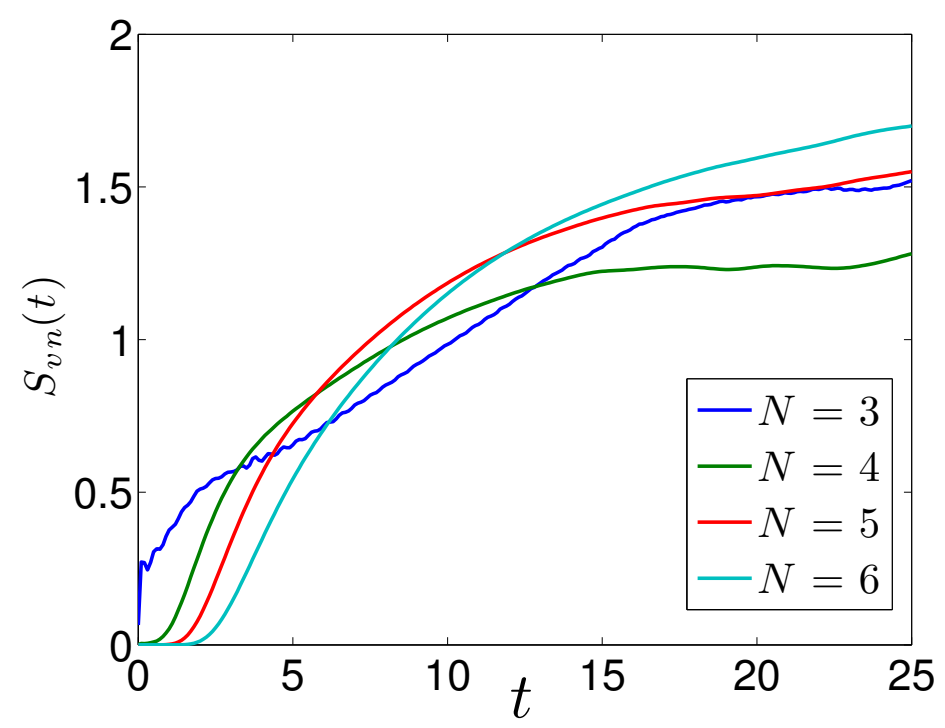

Figure 5. The time evolution of the entanglement entropy $S_{v n}(t)$ for the coherent state with a center at the centroid of the triangle is plotted for triangles with different $N$ by fixing the Planck constant $\hbar=0.025$.

In order to further explore the dependence of the quantum entanglement on the geometry of the triangular billiard, we compute the maximum value of the entanglement $S_{M}$ for different triangular configurations. In Figure $6 \mathrm{a}, \mathrm{b}$ we plot the maximum value of the entanglement entropy $S_{M}$ for different triangle configurations. In both figures (Figure 6a,b), blue lines show the entanglement maxima for the coherent states with $\hbar=0.1$ and $\hbar=0.025$ respectively. Similarly the black line shows the entanglement maxima $S_{M}$ for the negatively squeezed state and the red line shows the positively squeezed state. In our definition of the wave function, the negatively squeezed state gives a wave packet spread in the positions coordinates, hence it collides with the walls of the triangular billiard much faster and the entanglement develop much earlier and it gives a higher entanglement maxima. According to our definition of the wave packet, the positively squeezed state is highly localized in the position space and the wave packet takes more time to collide with the walls of the triangle and it shows a smaller entanglement entropy. From Figure $6 \mathrm{~b}$ it can be easily seen that, for the coherent state in the semiclassical limit, we get the similar result obtained for the irrationality of the triangle with the ground state eigenfunctions. It can be seen that the entanglement entropy is higher for a triangular configuration with $N$ closer to 10 . The entanglement maxima also shows the dependence on the irrationality of the triangle. We can also see that the squeezing can enhance or reduce the entanglement which depends on the sign of the quantum squeezing or in other words, it depends on whether we localize the wave function or spread it in the position coordinate. 


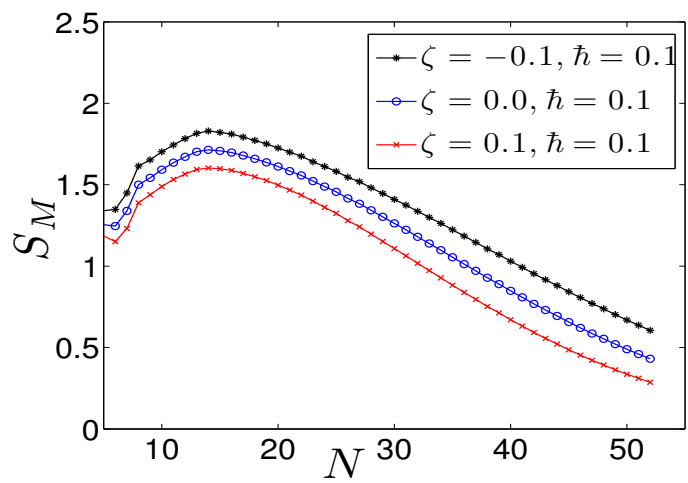

(a)

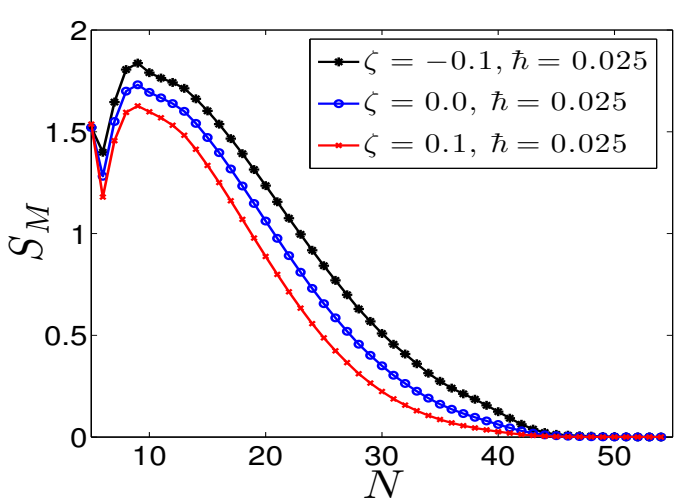

(b)

Figure 6. In (a) the maxima of von Neumann entropy of entanglement $S_{M}$ for the squeezed coherent state chosen at the centroid of the irrational triangles is plotted against the different triangle configurations with $\hbar=0.1$. In (b) the maxima of von Neumann entropy of entanglement $S_{M}$ for the squeezed coherent state chosen at the centroid of the irrational triangles is plotted against the different triangle configurations with $\hbar=0.025$. It can be easily seen that in both cases the entanglement entropy reduces as we increase $N$ or as we approach the equilateral triangle.

\section{Conclusions}

We have seen that the ground state eigenfunction entanglement reduces as we change the geometry from an irrational Pythagorean triangle to an equilateral triangle. We have also seen that the average entanglement of the first 500 eigenfunctions also changes as we change the geometry of the triangle and it is closely related to the irrationality of the triangle. We have also seen that the entanglement dynamics of the coherent state shows a clear dependence on the geometry of the triangle. It can also be seen that the quantum squeezing can be utilize to enhance or decrease the entanglement entropy in a triangular billiard.

Acknowledgments: This work was supported by the Spanish Ministry of Economy and Competitiveness under project number FIS2013-40653-P.

Author Contributions: Sijo K. Joseph and Miguel A. F. Sanjuán conceived and designed the problem and both of them equally contributed to the article. Both authors have read and approved the final manuscript.

Conflicts of Interest: The authors declare no conflict of interest.

\section{References}

1. Robnik, M. Classical dynamics of a family of billiards with analytic boundaries. J. Phys. A Math. Gen. 1983, 16, doi:10.1088/0305-4470/16/17/014.

2. Robnik, M. Quantising a generic family of billiards with analytic boundaries. J. Phys. A Math. Gen. 1984, 17, doi:10.1088/0305-4470/17/5/027.

3. Bunimovich, L. On the ergodic properties of nowhere dispersing billiards. Commun. Math. Phys. 1979, 65, 295-312.

4. Heller, E.J. Bound-State Eigenfunctions of Classically Chaotic Hamiltonian Systems: Scars of Periodic Orbits. Phys. Rev. Lett. 1984, 53, 1515-1518.

5. Berry, M.V.; Wilkinson, M. Diabolical Points in the Spectra of Triangles. Proc. R. Soc. Lond. A 1984, 392, $15-43$.

6. Artuso, R.; Casati, G.; Guarneri, I. Numerical study on ergodic properties of triangular billiards. Phys. Rev. E 1997, 55, doi:10.1103/PhysRevE.55.6384.

7. Casati, G.; Prosen, T. Mixing Property of Triangular Billiards. Phys. Rev. Lett. 1999, 83, doi:10.1103/PhysRevLett.83.4729. 
8. De Aguiar, F.M. Quantum properties of irrational triangular billiards. Phys. Rev. E 2008, 77, doi:10.1103/PhysRevE.77.036201.

9. Lima, T.A.; Rodríguez-Pérez, S.; de Aguiar, F.M. Ergodicity and quantum correlations in irrational triangular billiards. Phys. Rev. E 2013, 87, doi:10.1103/PhysRevE.87.062902.

10. Krishnamurthy, H.R.; Mani, H.S.; Verma, H.C. Exact solution of the Schrödinger equation for a particle in a tetrahedral box. Phys. A Math. Gen. 1982, 15, 2131-2137.

11. Aguirre, J.; Vallejo, J.C.; Sanjuán, M.A.F. Wada basins and chaotic invariant sets in the Hénon-Heiles system. Phys. Rev. E 2001, 64, doi:10.1103/PhysRevE.64.066208.

12. Lloyd, S.; Braunstein, S.L. Quantum computation over continuous variables. Phys. Rev. Lett. 1999, 82, 1784-1787.

13. Braunstein, S.L.; van Loock, P. Quantum information with continuous variables. Rev. Mod. Phys. 2005, 77, doi:10.1103/RevModPhys.77.513.

14. Andersen, U.L.; Leuchs, G.; Silberhorn, C. Continuous-variable quantum information processing. Laser Photonics Rev. 2010, 4, 337-354.

15. Weedbrook, C.; Pirandola, S.; Garcia-Patron, R.; Cerf, N.J.; Ralph, T.C.; Shapiro, J.H.; Lloyd, S. Gaussian quantum information. Rev. Mod. Phys. 2012, 84, doi:10.1103/RevModPhys.84.621.

16. Joseph, S.K.; Chew, L.Y.; Sanjuán, M.A.F. Impact of quantum-classical correspondence on entanglement enhancement by single-mode squeezing. Phys. Lett. A 2014, 378, 2603-2610.

17. Chaudhury, S.; Smith, A.; Anderson, B.E.; Ghose, S.; Jessen, P.S. Quantum signs of chaos in a kicked top. Nature 2009, 461, 768-771.

18. Ghose, S.; Sanders, B.C. Entanglement dynamics in chaotic systems. Phys. Rev. A 2004, 70, doi:10.1103/PhysRevA.70.062315.

19. Wang, X.; Ghose, S.; Sanders, B.C.; Hu, B. Entanglement as a signature of quantum chaos. Phys. Rev. E 2004, 70, doi:10.1103/PhysRevE.70.016217.

20. Joseph, S.K.; Sabuco, J.; Chew, L.Y.; Sanjuán, M.A.F. Effect of geometry on the classical entanglement in a chaotic optical fiber. Opt. Express 2015, 23, 32191-32201.

21. Varona, J.L. Rational values of the arccosine function. Cent. Eur. J. Math. 2006, 4, 319-322.

22. Eltschka, C.; Siewert, J. Quantifying entanglement resources. J. Phys. A Math. Theor. 2014, 47, 424005.

23. Berkovitza, J.; Friggb, R.; Kronzc, F. The ergodic hierarchy, randomness and Hamiltonian chaos. Stud. Hist. Phil. Mod. Phys. 2006, 37, 661-691.

24. Doya, V.; Legrand, O.; Mortessagne, F.; Miniatura, C. Speckle statistics in a chaotic multimode fiber. Phys. Rev. E 2002, 65, 056223.

25. Doya, V.; Legrand, O.; Michel, C.; Mortessagne, F. Optical scar in a chaotic fiber. Eur. Phys. J. Spec. Top. 2007, 145, 49-61.

26. Hollenhorst, J.N. Quantum limits on resonant-mass gravitational-radiation detectors. Phys. Rev. D 1979, 19, 1669.

27. Rai, J.; Mehta, C.L. Coordinate representation of squeezed states. Phys. Rev. A 1988, 37, doi:10.1103/PhysRevA.37.4497.

28. Caves, C.M. Quantum-mechanical noise in an interferometer. Phys. Rev. D 1981, 23, doi:10.1103/PhysRevD.23.1693.

29. Møller, K.B.; Jørgensen, T.G.; Dahl, J.P. Displaced squeezed number states: Position space representation, inner product, and some applications. Phys. Rev. A 1996, 54, doi:10.1103/PhysRevA.54.5378.

(C) 2016 by the authors; licensee MDPI, Basel, Switzerland. This article is an open access article distributed under the terms and conditions of the Creative Commons by Attribution (CC-BY) license (http:/ / creativecommons.org/licenses/by/4.0/). 